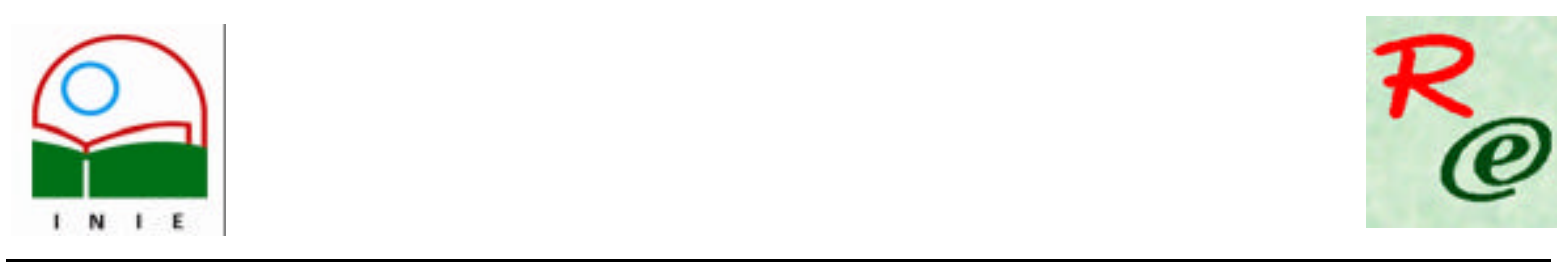

Actualidades Investigativas en Educación

Revista Electrónica publicada por el

Instituto de Investigación en Educación

Universidad de Costa Rica

ISSN 1409-4703

http://revista.inie.ucr.ac.cr

COSTA RICA

\title{
LAS GIRAS: UNA PRÁCTICA PEDAGÓGICA EN EL REPERTORIO PROBLEMAS ECOLÓGICOS, ESCUELA DE ESTUDIOS GENERALES, EN LA UNIVERSIDAD DE COSTA RICA
}

THE FIELD TRIP STUDY: A PEDAGOGICAL PRACTICE IN THE REPERTOIRE OF ENVIRONMENTAL PROBLEMS, SCHOOL OF GENERAL STUDIES, AT UNIVERSITY OF COSTA RICA

Volumen 9, Número 2

pp. $1-29$

Este número se publicó el 30 de agosto 2009

Ana Patricia Jiménez Morales

La revista está indexada en los directorios:

LATINDEX,$\underline{\text { REDALYC }}$ IRESIE, $\underline{\text { CLASE}}$, DIALNET, DOAJ, E-REVIST@S,

La revista está incluida en los sitios:

REDIE, RINACE, OEI, MAESTROTECA, PREAL, HUASCARAN, CLASCO 


\title{
LAS GIRAS: UNA PRÁCTICA PEDAGÓGICA EN EL REPERTORIO PROBLEMAS ECOLÓGICOS, ESCUELA DE ESTUDIOS GENERALES, EN LA UNIVERSIDAD DE COSTA RICA \\ THE FIELD TRIP STUDY: A PEDAGOGICAL PRACTICE IN THE REPERTOIRE OF ENVIRONMENTAL PROBLEMS, SCHOOL OF GENERAL STUDIES, AT UNIVERSITY OF COSTARICA
}

\begin{abstract}
Ana Patricia Jiménez Morales ${ }^{1}$
Resumen: Durante el primer semestre del 2007, se realizó un estudio para evaluar la opinión de los estudiantes del Repertorio de Problemas Ecológicos, de la Escuela de Estudios Generales de la Universidad de Costa Rica, 155 estudiantes fueron entrevistados después de participar en una gira de estudio como una actividad del curso. El objetivo de la investigación fue indagar la importancia de las giras al campo como apoyo a su formación profesional, así como promotora de un cambio de concepción de mundo en su relación con el ambiente. Además, era de interés evaluar la presencia del curso en el ámbito universitario debido a que es un curso de repertorio dirigido a estudiante de carreras diferentes a las ciencias básicas. Como resultado de la investigación se obtuvo que la mayoría de los entrevistados (70.97\%) ve la gira importante en su formación profesional, pues les permite una educación integral. Además, esta práctica le permitió al $65.8 \%$ de los participantes cambiar su concepción de mundo en relación con el ambiente y a un 19.35\% reafirmar sus creencias, permitiéndoles observar personalmente lo que ya conocían en forma teórica. A nivel Uhiversitario se comprobó que el curso tiene una presencia importante, los estudiantes que participaron como informantes pertenecían a 44 carreras de 12 facultades distintas.
\end{abstract}

Palabras clave: GIRAS DE ESTUDIO, EDUCACIÓN AMBIENTAL, PROBLEMÁTICA AMBIENTAL.

\begin{abstract}
During the first term of 2007 a study to evaluate the opinion of the students of the Repertoire of Ecological Problems, of the School of General Studies of the University of Costa Rica was carried out, 155 students were interviewed after participating in a study field trip. The objective of the research was to investigate the relevance of this practice in its professional development, as well as promotional of a change of world conception in its relation with the environment. In addition, it was of interest to evaluate the presence of the course in the university scope because it is a course of repertoire directed to student of careers different from basic sciences. As result was obtained that most of them (70. 97\%) considered that it was of much benefit in their professional development. The $65.81 \%$ who indicated this trip had motivated them to change their thinking with respect to the Nature, whereas $19.35 \%$ affirmed that this pedagogical practice had reaffirmed them in their commitment to the environment, being allowed them to observe personally what they already knew in theoretical form. It was determined that the course is very important in the university scope, since 44 careers of 12 different faculties were represented between the students participating in the study.
\end{abstract}

Key words: STUDY FIELD TRIPS, ENVIRONMENTAL EDUCATION, ENVIRONMENTAL PROBLEMS.

\begin{abstract}
TMáster en Biología de la Universidad de Costa Rica (UCR). Máster en Acuacultura de la State University of Ghent (RUG), Bélgica. Licenciada en Psicopedagogía de la Universidad Nacional de Costa Rica (UNA). Desde el 2000 ha sido Docente de la Sección de Ciencias Biológicas de la Escuela de Estudios Generales, Universidad de Costa Rica, impartiendo los cursos de Fundamentos de Biología, Problemas Ecológicos, Seminario Participativo de Humanidades, Seminario de Realidad Nacional y el de Hidroponía para el Programa de Cursos Libres. Ha sido Profesora de Introducción a la Problemática Ambiental y Didáctica Ambiental en la Dirección de Extensión Universitaria de la Vicerrectoría Académica de la Universidad Estatal a Distancia de Costa Rica (UNED).
\end{abstract}

Dirección electrónica: apjm21@yahoo.es

Artículo recibido: 8 de diciembre, 2008

Aprobado:24 de aaosto. 2009

Volumen 9, Número 2, Año 2009, ISSN 1409-4703 


\section{Introducción}

Según Solano-Núñez (2006), existe gran preocupación por los crecientes efectos adversos que tanto el desarrollo de la economía occidental como de la global ejercen sobre los recursos naturales. El daño a la Naturaleza es irreversible, algunas de las medidas tomadas por naciones apenas sirven para paliar la crisis que afecta sus recursos.

Es necesario realizar esfuerzos que permitan mejorar la calidad de vida de los ecosistemas y por ende de la humanidad, ya que somos un elemento más del ecosistema llamado "El Planeta Tierra". Para que ocurra lo anterior se requiere hacer conciencia entre las personas informándolas a cerca de las acciones que favorecen este deterioro, cuál es el estado actual de la crisis, y de si ya se ha llegado al punto de no retorno o eventualmente cómo se puede detener.

La Educación Ambiental como herramienta facilita el acceso de esa información a toda la ciudadanía. Contempla varios aspectos, no solo la enseñanza de la ecología, sino también la investigación de factores sociales, económicos y culturales que juegan un papel determinante en el manejo actual de cualquier recurso natural. (Gastreich, 2001). Dicha Educación permite

la formación de los individuos para conocer y reconocer las interacciones entre lo que hay de natural y de social en su entorno; y, para actuar en su entorno intentando no deteriorar el equilibrio que los procesos naturales han desarrollado, tendiente a lograr una calidad idónea para el desarrollo de la vida humana (Mata et al., 2003 p. 25).

Desde Rousseau (1712-1778), se concibió la Naturaleza como instrumento didáctico. Él utiliza el término naturaleza como el deseo explícito de educar utilizando el medio natural como una fuente de conocimiento y formación para los niños; no basta con enseñar desde la naturaleza utilizándola como recurso educativo, hay que educar para el medio ambiente, hay que presentar y aprender conductas correctas hacia el entorno, no solo conocerlo (SolanoNúñez, 2006).

Una herramienta de la metodología utilizada por la Educación Ambiental para cumplir con sus objetivos es la visita guiada al campo, en la que se expone al discente a una realidad, que en la mayoría de los casos ha sido referida en el salón de clase. Esta experiencia le Volumen 9, Número 2, Año 2009, ISSN 1409-4703 
permite desarrollar la observación, reflexionar sobre lo observado, analizar, trabajar en grupo, hacerse invisible en la naturaleza, pues aprenden a caminar sin hacer ruido, a escuchar los sonidos de la naturaleza, que generalmente están enmascarados por la contaminación sónica. Aún la visita a un lugar común, toma otro significado cuando se les enseña a volverse extranjeros en su propio entorno, pues aprenden a reconocer y entender su entorno de otra manera. De acuerdo con el constructivismo, que busca que el estudiante construya su propio conocimiento relacionándolo con su entorno y logrando alcanzar un aprendizaje significativo, es que las salidas al campo son muy utilizadas en las Ciencias Naturales pues permiten alcanzar estos objetivos.

La gira es de mucho valor, en especial cuando se usa con estudiantes de carreras que que tradicionalmente no son las que se relacionan con la Naturaleza y su protección, como Derecho o Economía, porque los sensibiliza de manera que su actitud ante los recursos naturales cambia.

Aquí es donde la presente investigación adquiere relevancia, pues evalúa la pertinencia de una gira de estudio utilizada como herramienta pedagógica de la Educación Ambiental que reafirma los fundamentos ecológicos discutidos en clase y permite visualizar los diferentes problemas ambientales directamente en el campo. Un aspecto de interés también es que permite identificar la presencia del curso Problemas Ecológicos en el ámbito universitario. Los objetivos del estudio fueron:

\section{Objetivos Generales}

- Evaluar la pertinencia de una gira al campo como estrategia pedagógica de la Educación Ambiental, que promueva en los y las estudiantes un cambio de actitud en su relación con el ambiente natural.

- Valorar la importancia del Repertorio de Problemas Ecológicos en el ámbito universitario.

\section{Objetivos Específicos}

- Determinar la opinión de los estudiantes del curso de Problemas Ecológicos sobre la utilidad de las giras de campo como práctica pedagógica que apoya su formación profesional. 
- Investigar la percepción de los discentes en relación con la gira como precursora de un cambio en su concepción de mundo acerca del ambiente.

- Analizar la presencia de un curso como el de Problemas Ecológicos en las diferentes Facultades y las carreras de la Universidad de Costa Rica.

\section{Metodología}

El Repertorio de Problemas Ecológicos en la Escuela de Estudios Generales de la Universidad de Costa Rica (UCR) se imparte bajo dos modalidades ${ }^{2}$ (opción seminario y opción regular). La primera asociada con el Seminario Participativo de Humanidades como cuarta materia en ella se trabajan dos grupos juntos de 40 estudiantes cada uno (80 en total) en una misma aula con cuatro docentes simultáneos, a saber de Filosofía, Comunicación y Lenguaje, Historia, y en nuestro caso, Problemas Ecológicos. La otra modalidad es un curso formal de Problemas Ecológicos en la que se atienden 30 estudiantes y hay un solo profesor o profesora.

En el primer semestre del 2007, la Sección de Ciencias Biológicas impartió cuatro grupos del repertorio en opción seminario y 9 grupos en opción regular, en los que se atendió, según la Oficina de Registro, a 430 estudiantes. De la población anterior se analizaron seis grupos, cuatro de la opción Seminario y dos de opción regular. Debido a que los grupos de seminario trabajan en parejas, para efectos del estudio, se considerará a cada pareja como uno solo, a pesar de que administrativamente son independientes.

Los grupos analizados fueron escogidos porque realizaron giras de estudio con el fin de reafirmar los conocimientos discutidos en el aula. Entre los objetivos perseguidos con una gira están enfrentar al y la estudiante con la realidad nacional, no solo ambiental sino social y económica, pues no puede aislarse el ambiente de estos otros aspectos. Durante las visitas al campo se promueven valores de respeto a la naturaleza y convivencia con sus pares, se

${ }^{2}$ Opción Seminario participativo es un curso integrado de Humanidades en el que se discute una temática desde la perspectiva humanística. Incluye cuatro materias simultáneamente; Filosofía, Historia de la Cultura, Comunicación y una cuarta materia que va a depender de la temática propuesta. En nuestro caso la temática es el medio ambiente por lo que esta cuarta materia es Problemas Ecológicos. La opción regular es un curso de repertorio en el que se discute la Problemática Ambiental, diseñados ambos para estudiantes de áreas de ciencias no básicas (Leyes, Ciencias Sociales, Ciencias Económicas, etc.) de manera que se complete su formación en forma integral al finalizar su carrera. 
les enseña a caminar por el bosque en silencio para no asustar a bs animales y poder escuchar los sonidos del bosque, el sonido del aire y los ruidos producidos por los diferentes organismos, los cuales en la ciudad están enmascarados por la contaminación sónica que nos rodea. Una práctica común utilizada en el laboratorio de campo es la observación, se le pide a los estudiantes que observen detenidamente a su alrededor mientras viajamos desde la Universidad hasta el primer punto de visita. A medida que transcurre el recorrido observan e identifican posibles áreas de riesgo de deslizamiento, problemas ambientales y sociales que tengan repercusión en el entorno natural como es la tugurización, mal manejo de los desechos sólidos, hacinamiento urbanístico, contaminación sónica, deforestación y contaminación de aguas entre otros. Una vez llegado al lugar de la primera visita se hace una puesta en común, en la que los y las estudiantes exponen sus observaciones y las discuten entre ellos, el o la docente actúa como moderador y al final hace un resumen de las observaciones aportadas y de las conclusiones obtenidas.

Cuando se visita un bosque se repasan conceptos ecológicos teóricos discutidos en clase como por ejemplo el concepto de ecosistema, componentes bióticos y abióticos - de manera que el bosque se convierte en una biblioteca abierta que permite a los jóvenes ver y comprender las diferentes relaciones ecológicas estudiadas, tanto intraespecíficas (por ejemplo depredación, y parasitismo) como intraespecíficas (reproducción, y división del trabajo entre otras), convirtiéndose la práctica en una herramienta útil que facilita al estudiante acomodar el nuevo conocimiento en su estructura cognitiva, favoreciendo de esta manera un aprendizaje significativo.

Al inicio de la gira se les entrega una guía con instrucciones claras, los objetivos por alcanzar y las actividades por realizar durante la visita -estos van a variar en función del lugar visitadose les indica lo que deben observar y analizar durante el viaje, así como el comportamiento que deben exhibir mientras estén en el autobús y dentro del bosque, puesto que es una clase formal al aire libre y se deben guardar la normas de comportamiento adecuados para cualquier casa de enseñanza.

Durante los recorridos se dan explicaciones de los aspectos relevantes observados, se hacen preguntas generadoras y se contestan las dudas de los y las estudiantes; también se interactúa con el personal del área visitada, por ejemplo guarda parques, administradores del Volumen 9, Número 2, Año 2009, ISSN 1409-4703 
lugar, educadores ambientales etc., que en ocasiones nos ofrecen una charla introductoria al lugar o nos acompañan a los recorridos.

Los resultados de la gira y su análisis se entregan por escrito en grupos de cuatro personas una semana después de realizada la misma, como representación de otra forma de aprendizaje en la que los miembros del grupo discuten sus observaciones por separado y las unen en un análisis conjunto, el cual es apoyado por información bibliográfica.

Para cumplir con los objetivos de la investigación a cada estudiante se le pidió indicar la carrera que cursaba y se le hicieron las siguientes preguntas que deberían responder por escrito y en forma individual.

1. ¿Considera usted que las giras realizadas tuvieron algún provecho para su formación profesional?

2. ¿Cree que le permitieron cambiar su concepción de mundo en relación con el ambiente?

Los grupos 1 y 2 corresponderán a opción seminario y el 3 y el 4 a opción regular. El grupo 1 realizó una gira al Parque Nacional Braulio Carrillo $^{3}$ y contestaron las preguntas 40 estudiantes. El grupo 2 visitó la Finca el Rodeo de la Universidad para la $\mathrm{Paz}^{4}$ y contestaron las preguntas 68 estudiantes. El grupo 3 realizó dos giras: una al Parque Nacional Tapantí

${ }^{3}$ Parque Nacional Braulio Carrillo. El Parque Nacional Braulio Carrillo es uno de los parques más extensos de Costa Rica, no solo cubre una extensión superior a las 44,099 hectáreas, sino que también alberga siete hábitats diferentes. Creado en 1978 para salvaguardar la diversidad de plantas y vida salvaje de la región. Permite estudiar durante su visita conceptos como nicho, hábitat, relaciones inter e intraespecíficas, adaptaciones de los organismos al ecosistema, entre otros.

${ }^{4}$ La Universidad para la Paz (UPAZ) es una institución académica dependiente de la Organización de las Naciones Unidas. Fue creada en 1980 por la Asamblea General de la ONU y tiene su sede en Ciudad Colón, Costa Rica Posee un área de protección de bosque conocida como El Rodeo, donde se puede observar una importante variedad de especies vegetales. El recorrido desde la UCR hasta la UPaz permite a los estudiantes analizar una serie de problemas urbanos, como congestionamiento vial, contaminación sónica y por gases, mala planificación de la urbanización entre otros. Una vez llegados al lugar permite analizar la importancia de reservas privadas que ayudan en la protección de la diversidad biológica de nuestro país. 
Macizo de la Muerte ${ }^{5}$ y otra a la Finca Agroecológica La Esperanza del señor Anselmo Rodríguez en Paracito de Moravia ${ }^{6} ; 26$ estudiantes contestaron las preguntas. El grupo 4 visitó la Finca Agroecológica La Esperanza; 21 estudiantes contestaron las preguntas. La muestra btal fue de 155 estudiantes, el 36\% de la matrícula teórica, porcentaje que representaría una proporción mayor al final del semestre ya que durante el mismo algunos estudiantes retiran el curso por diversas razones.

Las áreas visitadas se escogieron debido a tres razones, dos de tipo pedagógicas y una de disponibilidad de tiempo, la primera es que presentan características naturales que complementan los conceptos ecológicos discutidos en clase previamente, la segunda que en el trayecto hacia ella y en el lugar propiamente permiten analizar diferentes problemas de tipo ambiental y social discutidos en el temario del curso. En el pasado realizábamos giras de un día, de 7 a.m. a 5 p.m., lo que permitía visitar otras áreas más alejadas y variadas de manera que los estudiantes pudieran contrastar ecosistemas, pero en los últimos años ha sido política de las autoridades de la Escuela de Estudios Generales restringir las salidas al campo al periodo de clase (4h), de manera que la gira no interfiera con el horario de otros cursos que tienen matriculados nuestros estudiantes, por tal razón se escogen lugares cercanos al campus universitario.

\section{Resultados y Discusión}

Un aspecto relevante por considerar son las estrategias pedagógicas aprovechadas en el curso de Repertorio de Problemas Ecológicos con el fin de facilitar el proceso de enseñanza-aprendizaje en los discentes, entre ellas los viajes al campo, los cuales

\footnotetext{
${ }^{5}$ Parque Nacional Tapantí-Macizo de la Muerte. Se encuentra 90 minutos de San José, rodeado por la Reserva Macho y cruzada por el río Grande de Orosí. El sector Tapantí visitado durante la gira es de suma importancia como recurso hídrico, 150 ríos nacen en este Parque, presenta una gran variedad de ecosistemas y dos zonas de vida principales, el bosque montano bajo y la selva premontano, así como bosque nuboso, por lo que es muy rico en biodiversidad. Es una de las zonas más lluviosas del país, con una precipitación que puede alcanzar hasta $8000 \mathrm{~mm}$.

${ }^{6}$ Finca Agroecológica La Esperanza, ubicada en el Cantón de Moravia, Distrito Paracito, Provincia de San José. Dedicada a la producción de ganado de leche orgánico, de lumbricompost, de biogás, de hortalizas orgánicas, Además, realiza Educación Ambiental, recibe estudiantes a los que se les explica todos los procesos amigables con el ambiente que se realizan en la finca. Posee una hectárea de bosque de protección en la que se pueden observar especies tanto de flora como de fauna y se estudian procesos ecológicos que han sido discutidos previamente en clase. 
favorecen procesos mentales como la observación, el diagnóstico, la toma de decisiones, el análisis, la medición y la síntesis (UNESCO-PNUMA, 1987). Al potenciar en ellos estos procesos mentales se facilita la aprehensión de conocimientos viviendo e interiorizando la problemática que ven, sienten y huelen. En esas visitas el estudiante se enfrenta a una realidad, confronta la clase teórica con la práctica, vivenciando los problemas ambientales, y les permite acomodar ese nuevo conocimiento en su estructura cognitiva, alcanzando así un estado cognitivo superior (Zona de Desarrollo Próximo).

Es curioso observar el cambio que ocurre entre los estudiantes después del regreso de un laboratorio de campo: son más sensibles y asertivos; ahí han tenido la oportunidad de asimilar todos esos conocimientos teóricos e incorporarlos a su nueva estructura cognitiva, de donde no van a salir pues el nuevo conocimiento ha sido vivenciado en el entorno sociocultural en el que se desenvuelven a diario. De acuerdo con FUNDECOR (2001, p. 6), "La gira de campo es también una excelente forma de estimular en los estudiantes una de las habilidades más importantes a la hora de comprender y vivir el desarrollo sostenible: la habilidad de hacer conexiones" Esto debido a que se han convertido en extranjeros en su entorno y han aprendido a visualizar la realidad con otros ojos; ahora observan, no solo ven, y al observar e impresionarse con esa realidad aceptan el reto de ser cada vez más parte de la solución y cada vez menos parte del problema.

Al analizar las respuestas de los estudiantes a las preguntas planteadas se encontraron aspectos interesantes que serán analizados a continuación.

\subsection{La visita al campo como apoyo de la formación profesional del estudiante}

Los estudiantes que asisten a nuestros repertorios son, en su mayoría de carreras pertenecientes a áreas de ciencias no básicas. En muchos de sus programas de estudio de no se discuten los distintos problemas que sufren los ecosistemas naturales y mucho menos las formas en que, durante el ejercicio de su profesión ellos mismos van a ser responsables de este deterioro en un futuro no muy lejano. El curso de Problemas Ecológicos se dirige a estudiantes interesados en áreas ajenas a las ciencias básicas con el fin de ampliar su formación integral en el campo de la ecología y el ambiente (Sección de Ciencias Biológicas, 2009). Es por esta razón que decidió evaluarse la percepción del estudiante en relación con la influencia que podría tener tal práctica en su formación profesional. 
De la pregunta ¿Considera usted que las giras realizadas tuvieron algún provecho en su formación profesional? Se obtuvieron los datos presentados en el cuadro 1, un $70.97 \%$ de los estudiantes contestaron que sí resultaba importante en su formación profesional, pues aunque no eran de una área de ciencias básicas, en su desempeño profesional tenían que tomar decisiones relevantes que podrían amenazar el medio ambiente y por ende la calidad de vida de los ecosistemas y la humanidad, mientras que un $35 \%$ de ellos consideran que no tiene ningún provecho para su formación profesional.

Cuadro 1. Distribución, en porcentaje, de estudiantes que respondieron a la pregunta ¿Considera usted que las giras realizadas tuvieron algún provecho para su formación profesional?

\begin{tabular}{|cc|c|c|c|c|c|}
\hline Grupo & \multicolumn{2}{|c|}{ Sí } & \multicolumn{2}{c|}{ No } & \multicolumn{2}{c|}{ No Responde } \\
\cline { 2 - 7 } & $\mathbf{N}^{0}$ est. & $\%$ & $\mathbf{N}^{0}$ est. & $\%$ & $\mathbf{N}^{\circ}$ est. & $\%$ \\
1 & 30 & 75 & 5 & 12.5 & 5 & 12.5 \\
\hline 2 & 42 & 61.76 & 21 & 30.88 & 5 & 7.35 \\
\hline 3 & 19 & 73.08 & 7 & 26.92 & 0 & 0 \\
\hline 4 & 19 & 90.46 & 2 & 9.52 & 0 & 0 \\
\hline Total & 110 & 70.97 & 35 & 22.58 & 10 & $6.45 \%$ \\
\hline
\end{tabular}

Al analizar las 110 respuestas afirmativas se desprende que pueden ordenarse en cuatro premisas que representan las razones principales que ellos consideran como válidas. Estas se discuten a continuación:

\subsubsection{Les permitió obtener una formación integral}

Los estudiantes consideran la visita al campo como una parte de gran valor en su formación integral como profesionales y como personas. "Si, ya que este tipo de actividades ayudan a lograr el objetivo de la UCR que es ofrecer una educación integ ral y no solo una carrera en específico" (Estudiante de Contaduría Pública, 2007). ${ }^{7}$

\footnotetext{
7 Todas las referencias de los comentarios de los estudiantes utilizadas se escriben en la forma en que ellos las escribieron, conservando redacción y ortografía originales. 
No importa cuál sea su carrera, tanto las giras como el curso de Problemas Ecológicos les permite tener un panorama amplio, que a largo plazo les facilitará la toma de decisiones en forma más madura y crítica. "Claro, siempre, para cualquier carrera es importante saber de todo, ser integral y formarse un criterio para todo" (Estudiante de Trabajo Social, 2007).

El estudio y el análisis de la problemática ambiental deben realizarse en una forma interdisciplinaria debido a que los problemas analizados en el curso no son aislados. La crisis ambiental repercute en todos los niveles de la sociedad, en el ámbito social, económico, cultural y de salud. Debido a que el curso de Problemas Ecológicos se imparte a estudiantes de carreras como Derecho, Informática, Administración de Aduanas, entre otros, en el salón de clases se reúnen estudiantes de carreras diversas con un profesor o una profesora formados en biología, ese complemento permite discutir la problemática ambiental desde varios puntos de vista, complementando así la interdisciplinariedad perseguida, dándole un carácter holístico.

Por dicha razón, es necesario que todo profesional, sin importar cuál sea su orientación o su formación académica, esté conciente de esto, de manera que en su quehacer diario considere su entorno y evalúe las consecuencias de sus acciones, las cuales pueden tener un impacto positivo, pero que en muchos casos puede ser negativo. Por esto, como parte de la formación integral y humanista que pretende la Universidad de Costa Rica, un curso como el descrito es indispensable, ya que busca formar profesionales además de íntegros; responsables y sensibles ante su medio ambiente, acorde con los ideales del Humanismo pues como diría Don Rodrigo Facio $^{8}$ es necesario "preparar al joven en la "profesión del

\footnotetext{
${ }^{8}$ Don Rodrigo Facio Brenes (1917-1961) Catedrático de la Escuela de Derecho de Costa Rica y de la Escuela de Ciencias Económicas y Sociales, en la cual llegó a ser decano. Posteriormente desempeñó la Secretaría General de la Universidad de Costa Rica y luego fue Rector en varios períodos. Su labor es muy amplia y esta fue destacadísima no sólo en la enseñanza. En su gestión como rector de la Universidad de Costa Rica se le considera el reformador de la educación superior. Durante su administración se crearon los Estudios Generales, se hizo la reforma académica de la Facultad de Ciencias y Letras y la Universidad se trasladó a un nuevo Campus, donde se construyeron varios edificios. La Asamblea Universitaria, acordó denominar la Ciudad Universitaria, situada en San Pedro de Montes de Oca, Provincia de San José, "Ciudad Universitaria Rodrigo Facio Brenes" como justo homenaje a su memoria. La Asamblea Legislativa lo declaró Benemérito de la Patria por acuerdo № 397 del 22 de noviembre de 1961.
} 
hombre" antes de iniciar su preparación en las profesiones especializadas" (Soto, 2007. p38).

\subsubsection{Es un complemento práctico al conocimiento teórico}

Para los estudiantes visitar un área donde pueden observar una problemática ambiental es una experiencia sumamente enriquecedora que les permite complementar los conocimientos teóricos que obtiene en el aula, mediante las lecciones del profesor (a), los vídeos, conferencias y discusiones de clase. Esta práctica les permitirá a los estudiantes especialmente a aquellos que tienen un predominio visual en su aprendizaje- a aprehender esta información con mayor facilidad de manera que quedará en su estructura cognitiva para siempre, siendo accesible en un futuro cuando sea requerido de acuerdo con su experiencia profesional. "Si. Me parece que las giras ayudan mucho en la formación de una persona, es muy diferente ver algo teóricamente en la clase que realmente verlo / estudiarlo" (Estudiante de Ingeniaría Industrial, 2007).

Esta actividad les permite contrastar la teoría con la práctica, ya que para muchas personas es necesario vivenciar de manera práctica las problemáticas, favoreciendo una mayor sensibilización ambiental. "Las giras tuvieron una gran importancia, debido a que es la forma en que se ponen en práctica los conocimientos adquiridos en el aula, lo cual representa una muy buena forma de conocimiento "(Estudiante de Administración Pública, 2007). Las salidas de campo son una parte fundamental del trabajo del ecólogo en donde se ponen en práctica los conceptos y metodologías aprendidos en las clases, por lo que es importante que el y la estudiante la lleven a cabo como parte de su formación y para comprender mejor la problemática que nos rodea.

De acuerdo con FUNDECOR, las giras al campo desarrollan en el estudiante una actitud y postura más fuerte hacia el auto y co-aprendizaje, pues a través de ellas se logra demostrar que el conocimiento no está solo en los libros y en la mente del docente, sino en el ambiente, en las personas de la comunidad, en los hogares y especialmente en su capacidad y la de sus compañeros de buscar, sistematizar y utilizar ese conocimiento. (FUNDECOR, 2001, p. 6) 
Se busca con esta experiencia, basada en el modelo reflexión-acción, complementar la teoría con la práctica para que los estudiantes tengan una relación más armoniosa con la Naturaleza. Dicho modelo de investigación-acción es "un proceso cíclico en el cual la acción del maestro mejorada por la acción-reflexión, es vista como dialéctica entre teoría y práctica, tal como se ve el principio de relaciones recíprocas en Ecología" (UNESCO, 1994, citado por Solano-Muñoz, 200, p. 76).

Mediante la observación, el descubrimiento de hechos y el intercambio de opiniones con las personas (guarda parques, granjeros) que atienden en los lugares que visitamos, ellos construyen su propio conocimiento, se sensibilizan de acuerdo con las problemáticas analizadas, lo que lleva al fin último de la Educación, obtienen un aprendizaje significativo, pues el problema ya no es ajeno sino parte de su realidad, del contexto en el que se desarrollan diariamente. "Si porque permite ver en una forma práctica lo que en las aulas se estudia y permite dar una concepción más amplia" (Estudiante de Dirección de Empresas, 2007). Otro aspecto importante de confrontar la realidad es que les da acceso a nuevas herramientas para la resolución de problemas que enfrentarán en su práctica profesional, pues ven la realidad y cómo han resuelto situaciones los guarda parques, por ejemplo, conocer cómo ellos trabajan con mística por la protección del ambiente, en muchos casos motivados por el amor a la naturaleza, sin obtener nada a cambio, tan solo el placer de preservar las especies para que sus hijos tengan la oportunidad de disfrutarlas. "La carrera necesita no solo lo práctico, sino observar los problemas y soluciones que el hombre busca. El observar se interioriza mejor la necesidad de cuidar el ambiente. La clase en el campo es mejor" (Estudiante de Educación, 2007).

\subsubsection{Tiene aplicabilidad en su futura práctica profesional}

Un aspecto interesante de rescatar es que las respuestas se relacionan mucho con la carrera que cursa el estudiante, quienes analizan en qué podría favorecerlos tales experiencias. Unos, desde su perspectiva económica, comentan cómo va a ser su práctica profesional a partir de ahora, tratando de desarrollar su empresa en forma más amigable con el ambiente. "me parece que es de mucho provecho para mi formación profesional pues muestra una pequeña empresa que no provoca ningún deterioro ambiental, lo cual es algo importante para promover en nuestro país y en todo el planeta" (Estudiante de Dirección de Empresas, 2007). "Si porque mi 
carrera tiene que ver mucho con lo económico sin importarla naturaleza y en estas giras vi la realidad de Costa Rica y vi más la naturaleza que a la economía" (Estudiante de Contaduría Pública, 2007).

Por ejemplo, los estudiantes de agronomía encuentran en la visita a la finca agroecológica un ejemplo formidable de cómo aprovechar al máximo los recursos en una forma muy amigable con el ambiente y, más importante aún, que además sea rentable, ya que históricamente la práctica de la agricultura se ha visto como una forma de obtener vegetales comestibles, maximizando la producción en un menor tiempo posible, sin importar los impactos negativos que ocasionaba. Con estas prácticas se agotaron los suelos y para mantener ese nivel de producción se recurrió al uso de agroquímicos provocando una serie de problemas de salud en los ecosistemas y en la biota que se desarrolla en ellos. "Si me ayudó mucho, el hecho me dio una idea para cuando sea profesional, además es mucho provecho, ya que uno aprende mucho, se aprende más que en clase" (Estudiante de Agronomía, 2007). "Si, fue muy interesante y un complemento para la formación profesional y del curso" (Estudiante de Zootecnia, 2007). Este conocimiento no lo encuentran en los libros, ni en las aulas sino en la interacción con los propios actores.

\subsubsection{Facilita la obtención de una mayor conciencia ambiental y responsabilidad social}

Los estudiantes manifiestan que un aporte importante de la gira es que permite despertar una conciencia ambiental, conociendo la realidad que vive el país y de esta manera sensibilizarlos y motivarlos a dar soluciones a la problemática. Desde su perspectiva profesional ellos encuentran la relación del binomio ser humano-naturaleza con su desempeño futuro, lo que da una esperanza de que en su práctica ellos se vayan a detener un momento a pensar en el posible impacto ambiental y la forma de cómo minimizarlo. "Considero que al tratarse de conocer más del ambiente, y el medio con el que debemos desarrollarnos, es importante este tipo de actividades ya que sea cualquier carrera esta relación ser humano-naturaleza va más allá" (Estudiante de Trabajo Social, 2007).

Otro estudiante comenta: "Personalmente considero que ambas giras fueron de provecho para mí y parte importante de mi formación profesional, ya que me permitieron analizar y conocer un poco 
más sobre la realidad del deterioro ambiental de ello, en mi carrera (Dirección de Empresas) es muy importante este aspecto, pues para una organización es relevante llevar a cabo sus operaciones con responsabilidad social, sobre todo cuando se trata del ambiente y de su conservación, mas aun si tomamos en cuenta el deterioro que ocasionan las empresas que no cumplen con la normativa ambiental' (Estudiante de Dirección de Empresas, 2007).

Según el cuadro 1, 35 estudiantes $(22.58 \%)$ respondieron que consideraron que la gira no fue provechosa para su formación profesional; de estos 21 eran del grupo que visitó la Universidad para la Paz (UPaz). Las razones principales que argumentan fueron: lo corta que fue la gira, que era un área que no presentaba muchos problemas ambientales, el cansancio y el calor. "Faltó tiempo ya que fue muy rápido todo. Sin embargo no le encuentro relación con mi carrera" (Estudiante de Medicina, 2007). Valoraciones vacías que muestran la falta de sensibilidad ambiental y de interés por la actividad, la cual fue planeada con objetivos claros como observar durante el trayecto UCR - UPaz problemas ambientales como urbanización, manejo de desechos sólidos, contaminación sónica, entre otros; los cuales observamos todos los días de camino al trabajo o universidad, problemas tan familiares que ya se hacen invisibles ante nuestros ojos.

En sentido de mi formación profesional, creo que las giras no tuvieron gran impacto en mi carrera. Ya que la gira era referida o con sentido en la naturaleza y el ambiente, por lo que la aplicación del derecho no tiene mucho sentido que ver dentro de él. (Estudiante de Derecho, 2007).

Aquí denota falta de conocimiento de las diferentes especialidades del Derecho, siendo el derecho ambiental de sumo interés en la protección del medio ambiente. Para un profesional en el campo de las leyes es primordial sensibilizarse con la Naturaleza.

Para algunos de los estudiantes el lugar no fue el más apropiado, según ellos no había nada interesante, pero no son concientes que de que cualquier lugar que se escoja para una excursión, gira de estudio o laboratorio de campo, como quiera que se le llame tiene un valor didáctico intrínseco ya que: 
En una excursión se debe enfatizar la diferencia entre VER y OBSERVAR (...) mientras que el objetivo sea observar la excursión puede realizarse a lugares tan simples como áreas verdes de la escuela, el parque de la iglesia o el jardín de algún vecino (Brenes, 1999, p. 61)

El visitar el área protegida de la UPAZ les permitía conocer parte de la diversidad biológica que alberga nuestro país, el cual, según Obando (2002), es el más diverso del mundo, en términos de densidad de especies (№ de especies / unidad de área), por lo que era un lugar apropiado para conocer parte de esa diversidad biológica. Además, con una mentalidad más crítica y abierta hubieran analizado la importancia de una Reserva Privada, la cual coopera con la protección de esa diversidad genética que no solo es patrimonio de Costa Rica sino del mundo. La labor que realizan estas reservas privadas es primordial para la protección de nuestros recursos naturales, debido a que a pesar de que en teoría tenemos más de $25 \%$ del territorio nacional bajo alguna categoría de manejo (Parque Nacional, Reserva Biológica, etc.), la realidad es que el presupuesto que destina el Estado para su protección es insuficiente, por lo que muchos ecosistemas y especies en estas Áreas de Conservación se encuentran en peligro.

Manifestaciones como la siguiente mostraron que algunos estudiantes no lograron relacionar esta nueva información con su carrera, lo que podría ser por falta de madurez o desconocimiento de los alcances de su profesión futura, ya que consideran que sí fue útil para su cultura general, pero no para su vida profesional. Otra posible explicación a esta respuesta es que no internalizó que el lugar que estaba visitando es el último reducto de bosque primario de la Meseta Central, protegido por la Universidad para la Paz y es un sitio donde se ofrecen posgrados en diversas ramas del derecho.

No, para nada me pareció que fue una gran pérdida de tiempo poco provechosa y sin sentido. Lo único rescatable fue un poco de cultura general acerca de la Universidad de La Paz, el resto fue un paseo por un potrero. Disculpe la honestidad (Estudiante de Derecho, 2007)

Esto nos dice una vez más que no han visualizado aún que todos aquellos conocimientos que adquirimos en nuestras actividades diarias nos dan herramientas para resolver con un mejor criterio los retos a los que nos enfrentamos diariamente. Por ejemplo "La gira no tuvo Volumen 9, Número 2, Año 2009, ISSN 1409-4703 
gran impacto en los relacionado con la formación profesional" (Estudiante de Ingeniería Industrial, 2007) y "La gira fue muy corta y no hubo ninguna actividad provechosa para mi formación profesional." (Estudiante de Ingeniería Industrial, 2007). Estos estudiantes son de una carrera que impacta tanto el medio ambiente y no consideran la gira importante, posiblemente porque en su formación no hay concienciación del impacto que ellos son capaces de hacer con sus proyectos, lo que "da la impresión de que la importancia y trascendencia de la crisis ambiental siguen circunscribiéndose únicamente a ciertas áreas profesionales y que no interaccionan con la dinámica regular de la vida universitaria o lo hacen de manera muy precaria" (González-Gaudiano,1998 citado por Mata, 2003, p. 24). Tampoco son concientes aún de que existe toda una corriente de desarrollo amigable con el ambiente, que no lo limita sino que trata de reducir los impactos en el desempeño de su profesión. Pero opiniones como la siguiente nos dicen que aún quedan esperanzas para el medio ambiente.

"En realidad considero que no mucho con mi carrera, pero todo lo que lo haga a uno cambiar de parecer ver la realidad como realmente es y no como un adolescente, influye culturalmente sobre el pensar, lo que hace que uno vea la importancia de ser profesional y educarnos para ser mejor cada día" (Estudiante de Farmacia, 2007).

La cita anterior refuerza la necesidad de un curso en el que se enfrente al estudiante con otras disciplinas diferentes a su carrera de manera que su formación sea completa.

\subsection{La visita al campo como motivadora de un cambio de actitud en los estudiantes en relación con el respeto al ambiente}

La gira es un instrumento muy utilizado en Educación Ambiental que permite a las personas conocer de cerca los diferentes problemas, ese nuevo conocimiento les puede motivar a realizar cambios en su forma de pensar y ac tuar en relación con el ambiente, convirtiéndose en profesionales más responsables.

Se utilizó la pregunta ¿Cree que las giras le permitieron cambiar su concepción de mundo en relación con el ambiente? para determinar cuál era la creencia de los estudiantes con 
respecto a esto. ¿Les permitió cambiar su concepción de mundo? Sí, No o por el contrario ya se habían comprometido con el ambiente y su protección y la gira les permitió reafirmar ese compromiso.

El cuadro 2 nos muestra que el $65.81 \%$ de los estudiantes considera que la experiencia en el campo sí promovió un cambio en su concepción de mundo en relación con el ambiente, porque "este acercamiento me permitió observar y comprender muchas de las relaciones existentes en los bosques y las cuales no apreciaba de la misma forma" (Estudiante de Educación Primaria). Esta nueva forma de ver la naturaleza le ayudará en su formación; además, en el futuro esta visión será transmitida a las personas que conforman su núcleo familiar, laboral y social, convirtiéndose en un ente multiplicador de esa conciencia ambiental, lo que se traducirá en una mejor protección de los recursos naturales. "La gira si me cambio la concepción del ambiente y valorar las especies componentes del bosque que nos benefician a largo plazo" (Estudiante de Administración Aduanera, 2007). "Si, ya que al estar en contacto con la naturaleza uno se percata del daño al medio ambiente causado por el hombre lo cual nos hace tomar conciencia acerca de lo afortunado que somos al tener tan bello medio ambiente" (Estudiante de Administración Pública, 2007).

La experiencia, como se dijo antes, les permitió conocer realidades, observar y evaluar el impacto que provocamos con nuestras acciones, además conocieron personas comprometidas con la proteccion del ambiente. Todo esto tocó sus fibras y los hizo tomar conciencia del problema que enfrentamos y del dilema en el que vivimos, aceptamos nuestra responsabilidad y decidimos hacer un cambio o continuamos actuando irresponsablemente y nos hundimos con el planeta.

Un $10.97 \%$ considera que no hubo ningún cambio en su concepción de mundo en relación con el ambiente; ellos seguirán pensando del mismo modo. Es una lástima que estudiantes universitarios no hayan aprovechado la oportunidad que se les dió de tomar conciencia, la esperanza es que en un futuro cercano haya un evento importante en sus vidas que les permita hacerlo para que a partir de ese momento ejerzan sus profesiones en una forma responsable y no sea la Historia quien llegue a señalarlos. Estos comentarios serán tomados en cuenta en la planificación de giras y actividades del próximo curso, de manera 
que se le preste atención a las creencias, actitudes y comportamientos del estudiantado desde el inicio.

Un $19.35 \%$ considera que la actividad les permitió reafirmar sus valores y compromiso con el medio que les rodea, porque ya sentían respeto por la naturaleza y que la gira les había permitido fortalecer aún más esa conciencia ambiental. "No, por el contrario confirmaron lo adquirido con anterioridad así como valores personales en relación con el ambiente" (Estudiante de Enseñanza del Inglés, 2007). "Considero haber tenido siempre una concepción del mundo y del ambiente correctos" (Estudiante de Artes Dramáticas, 2007). Esto es un estímulo que demuestra que el Sistema Educativo nuestro, en alguna medida, está contribuyendo a mejorar el Planeta por medio de sus Programas en los que en forma transversal se discute la problemática ambiental en todas las materias, ya que en Costa Rica el Ministerio de Educación Pública ha elaborado planes y programas de enseñanza en los que incorpora la Educación Ambiental como un componente más en el currículo nacional. (Solano, 2006). Gracias a este interés por fomentar el respeto por su entorno estos estudiantes son personas concientes de su realidad y sensibles ante los cambios que sufre el Planeta y la gira para ellos no fue más que la confirmación de lo que ya sabían "el Planeta está en crisis, está muriendo y con él moriremos nosotros".

En resumen, más del 85\% de los entrevistados (los que contestaron Sí y los que reafirmaron sus creencias) tendrán una mejor actitud en su actuar diario, en relación con la salud del Planeta; como resultado de una vista al campo ellos serán ambientalmente más responsables.

\section{Cuadro 2. Distribución de estudiantes que respondieron a la pregunta ¿Cree que las} giras le permitieron cambiar su concepción de mundo en relación con el ambiente?

\begin{tabular}{|c|c|c|c|c|c|c|c|c|}
\multirow{2}{*}{ Grupo } & \multicolumn{2}{|c|}{ Sí } & \multicolumn{2}{c|}{ No } & \multicolumn{2}{c|}{ Reafirma } & \multicolumn{2}{c|}{ No Responde } \\
\cline { 2 - 9 } & $\mathbf{N}^{0}$ est. & $\%$ & $\mathbf{N}^{0}$ est. & $\%$ & $\mathbf{N}^{0}$ est & $\%$ & $\mathbf{N}^{\circ}$ est. & $\%$ \\
\hline 1 & 30 & 75 & 3 & 7.5 & 7 & 17.5 & 0 & 0 \\
\hline 2 & 31 & 45.59 & 13 & 19.12 & 18 & 26.47 & 6 & 7.5 \\
\hline 3 & 25 & 96.15 & 1 & 3.83 & 0 & 0 & 0 & 0 \\
\hline 4 & 16 & 76.19 & 0 & 0 & 5 & 23.81 & 0 & 0 \\
\hline Total & 102 & 65.81 & 17 & 10.97 & 30 & 19.35 & 6 & 3.87 \\
\hline
\end{tabular}


De acuerdo con FUNDECOR (2001), la gira de campo es adecuada para cumplir con los objetivos de la Educación Ambiental, pues es flexible, abierta, sistemática y problematizadora. Las salidas al campo posibilitan el aprendizaje significativo en las Ciencias de la naturaleza y contribuyen a la educación ambiental de los alumnos (López ${ }^{9}, 2000$ ). Las giras de campo en muchos casos han sido vistas como inapropiadas en el proceso de enseñanza-aprendizaje y han tenido poco apoyo como práctica pedagógica, algunas de las razones válidas y otras no tan válidas de esto son por ejemplo aspectos económicos y la seguridad de los y las estudiantes, el miedo del profesorado a las responsabilidades que se asumen es este tipo de actividades o la falta de planificación y metodología adecuada lo que impide un buen aprovechamiento de la actividad. Pero esto debe cambiar pues hace que tanto docentes como estudiantes, pierdan una experiencia única de aprendizaje, la cual nosotros podemos disfrutar con facilidad, mientras que en otros países es difícil porque no cuentan con la riqueza natural que tenemos en Costa Rica.

La importancia de una gira o visita al campo se pone de manifiesto en los resultados aquí expuestos como una estrategia pedagógica ideal para el proceso de enseñanza aprendizaje de la Educación Ambiental, la cual "tiene como objetivo primordial formar personas capaces de comprender la problemática ambiental y motivados para participar en su resolución" (Gastreich, 2001, p. 9), contribuyendo así a fomentar responsabilidad ambiental en la población estudiantil, que va a ser en un futuro cercano la que tome las decisiones del país; decisiones que pueden llegar a representar la vida o muerte de todas las formas de vida del Planeta.

En un estudio anterior realizado por Amador (2003), no se analizó la pertinencia de una gira, sino del curso de Problemas Ecológicos como tal, la mayoría de los estudiantes (88\%) consideró que la temática discutida durante el semestre sería importante en su formación profesional y un $93 \%$ señaló que le había fomentado un cambio de actitud en relación con el medio que le rodea. Al ser una estrategia pedagógica utilizada para promover estos cambios, la gira resulta de mucha utilidad ya que les presenta la realidad en que viven de una forma amena lo que facilita en ellos el aprendizaje significativo. "Considero que la gira fue una

${ }^{9}$ Catedrático de Biología - Geología. IES Ramon Arcas Meca, Lorca. Universidad de Murcia, España. Consejería de Educación, Ciencia e Investigación. Dirección General de Promoción Educativa e Innovación. Volumen 9, Número 2, Año 2009, ISSN 1409-4703 
excelente práctica o actividad realizada en grupo. Añado también que fue muy entretenida y una experiencia agradable" (Estudiante de Farmacia, 2007).

La cita siguiente podría considerarse como un resultado relevante en la investigación, pues resume el sentir de los estudiantes con respecto a la experiencia de salir al campo y al hecho de analizar la problemática ambiental en un curso como el de Problemas Ecológicos.

"Después de analizar o recobrar un poco de conciencia sobre nuestra situación actual, gracias a las giras y a lo comentado en clase; y tratar de saber qué será de nosotros en un futuro si no cambiamos nuestra forma de pensar y actuar, en mi, personalmente crea un deseo de hacer algo no solo para mi, sino por mi familia, mi comunidad y mi planeta tierra, que al fin y al cabo es el lugar donde viviré el resto de mi vida y donde vivirán nuestros descendientes, y si en realidad deseamos vivir en armonía con la naturaleza, para así lograr que nuestros preciados recursos naturales sean conservados para nuestras futuras generaciones tenemos que comenzar a cambiar nosotros mismos para así luego tratar de incitar a las otras personas a que cuidemos lo nuestro y lo que sin ningún precio la madre tierra nos da" (Estudiante de Estadística, 2007).

\subsection{Importancia del curso de Problemas Ecológicos en el ámbito universitario}

El Repertorio de Problemas Ecológicos pretende dar bases ecológicas y sensibilizar a los estudiantes en la problemática ambiental de nuestro planeta, pero especialmente la de Costa Rica. También es su objetivo completar su formación integral, facilitando de esta manera su inserción crítica en la realidad histórica en que viven. En este curso se estudian los problemas ambientales que provocan los procesos de industrialización y el incremento de la población entre otros, como por ejemplo: el urbanismo, la pérdida de especies, la contaminación en sus diferentes formas, etc.

Los cuadros 3 y 4 muestran la distribución del total de estudiantes, por facultades y por carreras, que participaron en giras de estudio en los gupos analizados durante el primer semestre del 2007. 
Cuadro 3. Distribución por Facultades de estudiantes participantes en los grupos de Problemas Ecológicos analizados.

\begin{tabular}{|l|c|c|}
\hline \multicolumn{1}{|c|}{ FACULTAD } & N N $^{\mathbf{c}}$ DE EST. & $\%$ \\
\hline Ingeniería & 39 & 25.16 \\
\hline Ciencias Económicas & 31 & 20 \\
\hline Medicina & 24 & 15.48 \\
\hline Ciencias Sociales & 16 & 10.32 \\
Ciencias de la Educación & 11 & 7.10 \\
\hline Derecho & 10 & 6.45 \\
\hline Letras & 5 & 3.23 \\
\hline Ciencias Agroalimentarias & 4 & 2.58 \\
\hline Ciencias & 4 & 2.58 \\
\hline Odontología & 4 & 2.58 \\
\hline Microbiología & 3 & 1.93 \\
\hline Bellas Artes & 1 & 0.64 \\
\hline No responde & 3 & 1.93 \\
\hline TOTALES & 155 & 100 \\
\hline
\end{tabular}

\section{Cuadro 4. Carreras representadas en cada Facultad en los grupos de Problemas} Ecológicos analizados.

\begin{tabular}{|l|l|}
\hline \multicolumn{1}{|c|}{ FACULTAD } & \multicolumn{1}{c|}{ CARRERAS } \\
Medicina & Nutrición, Farmacia, Medicina, Enfermería y Salud Pública. \\
\hline Microbiología & Microbiología \\
\hline Odontología & Odontología \\
\hline Ciencias Económicas & $\begin{array}{l}\text { Economía, Administración Aduanera, Ciencias Actuariales, Dirección de } \\
\text { Empresas, Administración Pública, Contaduría Pública y Estadística. }\end{array}$ \\
\hline Letras & Filosofía e Inglés. \\
\hline $\begin{array}{l}\text { Ciencias } \\
\text { de la Educación }\end{array}$ & $\begin{array}{l}\text { Enseñanza de los Estudios Sociales, Educación Primaria, Enseñanza del } \\
\text { Inglés, Enseñanza de la Filosofía, Enseñanza del Español y Preescolar. }\end{array}$ \\
\hline Ciencias & Meteorología y Química. \\
\hline Derecho & Derecho \\
\hline Ciencias Sociales & $\begin{array}{l}\text { Ciencias Políticas, Psicología, Historia, Antropología, Comunicación } \\
\text { Colectiva, Sociología y Trabajo Social. }\end{array}$ \\
Ing. Química, Ing. Eléctrica, Ing. Civil, Ing. Industrial, Ing. Mecánica, \\
Ingeniería & Arquitectura, Topografía, Ciencias de la Computación e informática. \\
\hline Ciencias Agroalimentarias & Zootecnia e Ingeniería Agrícola. \\
\hline Artes & Artes Plásticas (Pintura). \\
\hline
\end{tabular}


En los grupos analizados, que equivalen al $46 \%$ del total que se impartieron en el periodo de estudio, había estudiantes de 44 carreras distribuidos en 12 facultades. El mayor número de estudiantes pertenecían a las áreas de Ingeniería y de Ciencias Económicas con $25.16 \%$ y $20 \%$ respectivamente. Le seguía Medicina con un 15.48\%, los de Ciencias Sociales representaron un $10.32 \%$; las otras áreas como Derecho, Computación, Ciencias Agrarias, estuvieron representadas por menos de 10 estudiantes (6.45\%).

En el estudio de Amador (2003) el área predominante fue la de Ciencias Sociales con un $46 \%$ seguido por Ingeniería con un 32\%. Ciencias Económicas que en nuestro caso representa el segundo lugar con un $20 \%$ no aparece reportado en Amador (2003). Para las áreas de ciencias básicas y ciencias agroalimentarias el comportamiento de los resultados es similar en ambos estudios ( $2 \%$ y $3 \%$ respectivamente).

El número de carreras (44) representadas por los estudiantes que utilizaron el Repertorio de Problemas Ecológicos como curso de servicio es significativo, lo que muestra el alcance y relevancia del mismo en la Universidad. El trabajo en el curso que se realiza es significativo, siendo éste una labor prioritaria debido a la crisis ambiental que enfrentamos en el mundo. Se requiere de una Educación Ambiental continua dirigida tanto a niños como a jóvenes y adultos. El objetivo nuestro es el compromiso con las presentes y futuras generaciones, un compromiso que nos lleva a luchar por una mayor concienciación de la población que llega a nuestras aulas, en pro de una mejor salud ambiental para el Planeta.

En los grupos de opción seminario, la variabilidad de carreras representadas osciló entre 23 y 25, mientras que en los de opción regular varió de 13 a 15, lo que podría explicarse por el hecho de que el grupo 4 es de horario nocturno y lo matriculan principalmente estudiantes que trabajan durante el día y la mayoría son de áreas de Ciencias Económicas y Sociales; además, el número de estudiantes por grupo es menor que en los de opción seminario. Un aspecto interesante es que los estudiantes que matriculan nuestros cursos muestran una gran variedad de formas de pensamiento y puntos de vista que enriquecen las discusiones de clase, pues al tener tal diversidad de carreras representadas, igual de diversos son los aportes que ellos hacen, en especial con el enfoque que dan a sus investigaciones realizadas durante el semestre. Lo anterior permite que los temas que se discuten, aunque 
son comunes todos los años, se vean fortalecidos por la actualidad aportada en las discusiones.

Antes de concluir, es importante discutir un punto trascendental relacionado con las salidas al campo, ya sea en un curso de Problemas Ecológicos, como de Biología o Geología. La buena planificación de cualquier práctica pedagógica es necesaria para que sea exitosa y de provecho para el alumnado, y para una gira también. El o la docente deben conocer bien el sitio a visitar, buscar mapas y toda aquella información que pueda ser de utilidad para la consecución de los objetivos de la misma. Los estudiantes deben conocer y tener claros dichos objetivos y las actividades a realizar durante todo el trayecto, así como los puntos de interés a visitar. De más está decir que el lugar escogido debe presentar características que concuerden con aspectos previamente discutidos en clase de manera que la salida sea un complemento del temario a desarrollar durante el curso. Las actividades deben ser cuidadosamente planeadas para que los y las estudiantes sean actores en ese proceso de enseñanza-aprendizaje, que ellos puedan construir su propio conocimiento con el o la docente actuando como motivadores y acompañantes del proceso. De esta planificación dependerá el éxito de la gira. Es muy importante además observar comportamientos como el consumo de alimentos, conductas durante los recorridos y en el autobús para relacionarlos con lo que se observa durante el viaje.

\section{Conclusiones}

La mayoría de los estudiantes (70.97\%) considera que la gira fue fundamental en su educación profesional, pues es parte de esa formación integral que pretende la Universidad de Costa Rica dar a sus estudiantes. Además, son un complemento práctico del conocimiento teórico discutido en el salón de clase, permitiendo una mejor comprensión de los contenidos del programa (ver anexo). Los ejemplos observados en las salidas al campo y el contacto con otros profesionales o campesinos con los que tienen la oportunidad de conversar en las áreas visitadas permiten identificar aciertos y desaciertos en el manejo de los recursos naturales, también obtienen ideas para implementar en su futura práctica profesional, como es el caso de la finca orgánica visitada.

La gira es una práctica pedagógica apropiada para realizar Educación Ambiental ya que rompe con la rutina habitual del salón de clase y permite a los alumnos y alumnas vivenciar Volumen 9, Número 2, Año 2009, ISSN 1409-4703 
los problemas ambientales cotidianos en el entorno social del país. Les enseña a ver su entorno de otra manera más objetiva, aprender a observar e identificar problemas ambientales, aun en su ambiente cotidiano.

Las salidas al campo motivaron a los estudiantes a un cambio en su forma de pensar con respecto al ambiente, lo que se traducirá en una actitud y práctica profesional más responsable con el ambiente. Esta actividad les permitió adquirir una mayor conciencia ambiental o en su defecto reafirmar sus creencias en relación con la problemática ambiental que sufre nuestro país y la protección de los recursos naturales.

La importancia del curso de Problemas Ecológicos en la Universidad de Costa Rica queda manifiesta al tomar en cuenta el número de carreras (44) y facultades (12) que se encuentran representadas en los estudiantes entrevistados. Esto hace referencia al alcance de la labor de la concienciación ambiental que se realiza en el curso.

La salida al campo facilita el desarrollo de destrezas cognitivas de alto nivel como la observación, análisis y descubrimiento del medio natural, esto les motivará a ser más concientes de la problemática ambiental y la protección de medio que les rodea. Pero es importante tener en cuenta que la gira requiere de una planificación adecuada de las actividades a realizar por los y las estudiantes, la escogencia del lugar apropiado de acuerdo con los objetivos y además de suficiente motivación por parte del docente entre los educandos para asegurar su éxito.

El resultado de la investigación es un estímulo para hacer mejor nuestro trabajo, pues demuestra con un grado de confiabilidad que la Educación Ambiental que se realiza en el Repertorio de Problemas Ecológicos, utilizando la estrategia pedagógica de la visita al campo es apropiada, pues mediante una práctica agradable los estudiantes se enfrentan a la realidad nacional, lo que les permitió, como se dijo antes ser extranjeros en su propio entorno, haciendo significativo este nuevo conocimiento, de manera que será parte de su formación y les será útil en su vida profesional.

Para cerrar hago mías las palabras de López Martín (2007, p. 102) cuando dice "Querer enseñar geología sin salir al campo es como estudiar informática sin utilizar ordenadores", yo Volumen 9, Número 2, Año 2009, ISSN 1409-4703 
diría "Querer enseñar Problemas Ecológicos sin una gira para vivenciar la problemática directamente en el campo es como estudiar para ser chef y no tener una cocina"

\section{Agradecimientos}

Deseo agradecer muy especialmente a los estudiantes del curso Problemas Ecológicos que muy amablemente accedieran a participar en este estudio como informantes, a las profesoras del Curso de Problemas Ecológicos Lisbeth Araya, Sonia Ramírez y Lorena Chavarría que me permitieron pasar las encuestas en uno de sus grupos respectivamente y así completar la información recabada con los estudiantes de mi grupo. Por último a mis compañeros del la Sección de Comunicación Oscar Alvarado, Ricardo Vargas y Leda Cavallini quienes me hicieron el favor de enriquecer este manuscrito con sus atinadas observaciones y sugerencias filológicas.

\section{Referencias}

Amador Berrocal, Sonia María. (2003, abril). Elementos Biológicos como parte de la formación humanística. En Memoria Histórica del Congreso de humanidades: Cultura, Universidad y Humanismo en el Siglo XXI (pp. 203-207). San José, Costa Rica. Revista de la Escuela de Estudios Generales (número extraordinario), Universidad de Costa Rica.

Brenes, Olga Emilia. (1999). Estrategias Didácticas. San Pedro de Montes de Oca: EUNED.

Fundación para el desarrollo de la Cordillera Volcánica Central - FUNDECOR. (2001). Gira al Volcán Poás como experiencia educativa. Una propuesta de la Fundación para el desarrollo de la Cordillera Volcánica Central. San José, Costa Rica: FUNDECOR.

Gastreich, Karin R. (2001). Aprendamos en el aula verde. Laboratorios de campo en el Bosque Iluvioso, con información para facilitadores. San José, Costa Rica: Fundación Neotrópica. Editorial Heliconia.

López-Martín, Juan Antonio. (2007). Las salidas al campo: mucho más que una excursión. [Versión electrónica]. Revista Educar en el 2000: Revista de Educación ambiental (11), pp. 100-103. Murcia. España.

Mata Segreda, Alejandrina et al. (2003). Estrategias Innovadoras para la formulación inicial de educadores en el campo ambiental (Informe de Investigación). San José: 
Coordinación Educativa y Cultural Centroamericana. Consejo Nacional de Rectores. Comisión de Vicerrectores de Investigación. Subcomisión de Educación Ambiental.

Obando Acuña, Vilma. (2002). Biodiversidad en Costa Rica: estado del conocimiento y gestión. Heredia, Costa Rica: Instituto de Biodiversidad, INBio.

Universidad de Costa Rica. Escuela de Estudios Generales . (2009). Programa del Curso de Problemas Ecológicos. San José: Sección de Ciencias Biológicas.

Solano Muñoz, Edgar. (2006). La Evolución de la Educación Ambiental en Costa Rica. Revista de Ciencias Sociales 111-112, 71-80. Universidad de Costa Rica.

Soto Valverde, Gustavo Adolfo. (2007). 50 Aniversario de los Estudios Generales de la Universidad de Costa Rica. Documentos fundamentales (Edición Conmemorativa). San José, Costa Rica: Editorama

UNESCO-PNUMA. (1987). Enfoques pedagógicos a la Educación Ambiental. En Rodríguez Mayra, María Eugenia Zúñiga y Estrella Guier, Antología de Didáctica Ambiental (pp.121-145). San José, Costa Rica: EUNED. 


\section{Anexo}

Objetivos y contenidos discutidos en el curso de Problemas Ecológicos, tomados del Programa del 2009

\section{OBJETIVOS DEL CURSO}

\section{Objetivos generales}

-Revisar los principios generales de la ecología para analizar los problemas ecológicos más sobresalientes en la actualidad que afectan a las diferentes formas de vida.

-Conocer soluciones que se aplican para permitir un balance adecuado entre el uso inmediato y la protección de los recursos, con el fin de garantizar su disponibilidad, uso y disfrute para las generaciones futuras.

\section{Objetivos específicos}

-Explicar los fundamentos básicos de la ecología, para comprender e papel de los seres vivos y del medio ambiente.

-Analizar las relaciones existentes entre los constituyentes de los ecosistemas y su dinámica de adaptación, con la finalidad de entender el funcionamiento de la naturaleza.

-Examinar las características físicas y geológicas de Costa Rica que explican la biodiversidad tan abundante presente en el país.

-Analizar las diversas causas y consecuencias del desequilibrio ecológico del planeta y de Costa Rica en particular para estudiar casos específicos.

-Discutir formas de manejo en las asociaciones y ecosistemas del planeta para el posible restablecimiento del equilibrio. 
- Destacar la importancia de la investigación científica y tecnológica, de la educación y de la Legislación ambiental, para la protección y uso adecuado de los recursos naturales y del ambiente.

\section{CONTENIDOS}

\section{PARTE: PRINCIPIOS GENERALES DE ECOLOGÍA MODERNA}

\section{Introducción}

Importancia de la ecología como ciencia

Desarrollo histórico de la ecología y su relación con el humanismo

Conceptos generales de ecología moderna

\section{Constituyentes, relaciones y adaptaciones en el ecosistema}

Factores abióticos físicos y químicos.

Factores bióticos.

Dinámica de adaptación.

\section{Funcionamiento del ecosistema}

Ciclos biogeoquímicos.

Niveles de evolución.

Niveles de organización de la materia.

Flujo de materia-energía.

\section{Biodiversidad}

Diversidad biológica.

Sistema de zonas de vida.

Biodiversidad de Costa Rica.

\section{PARTE: SITUACIÓN ECOLÓGICAACTUAL}

\section{Bosques y áreas silvestres.}

Importancia.

Situación en Costa Rica.

Deterioro, causas, consecuencias y estrategias. 


\section{Sistemas agropecuarios.}

Impacto en el ambiente.

Problemática de los agroquímicos.

Alternativas a la agricultura convencional.

\section{Crecimiento demográfico y urbanización.}

Aumento de la población humana

Crecimiento del área urbanizada.

Efectos en el ambiente: disposición de desechos y problemática del agua.

\section{Situación atmosférica.}

Alteraciones del efecto invernadero.

Cambio climático global

Deterioro de la capa de ozono.

Lluvia ácida.

\section{Ecosistemas marinos.}

Constituyentes

Diversidad de ecosistemas

Recursos marinos

\section{Biotecnología, sus aplicaciones y repercusiones}

Procesos biotecnológicos

Aplicaciones ambientales

Impacto en los ecosistemas. 